Vol. 20(2011): 143-150.

\title{
Comparison of different DNA extraction methods from hair root follicles to genotype Finnish Landrace boars with the Illumina PorcineSNP60 BeadChip
}

Anu Sironen*, Pekka Uimari, Johanna Vilkki

MTT Agrifood Research Finland, Biotechnology and Food Research, FI-36100 Jokioinen, Finland

*email: anu.sironen@mtt.fi

Recent developments in sequencing methods have enabled whole genome sequencing of several species and the available sequence information has allowed the development of high throughput genotyping chips. However, these genotyping methods require high quality DNA. The possibility to genotype samples based on DNA from non-invasive sources would permit retrospective genotyping of previously collected samples and also facilitate the analysis of large populations e.g. for genomic selection. In this study we have developed and evaluated different DNA preparation methods from porcine hair root follicles for high throughput genotyping with the PorcineSNP60 Genotyping BeadChip (Illumina). We describe a method for DNA extraction from porcine hair root samples, which produces results from high throughput genotyping with the same high degree of accuracy as previously reported for DNA extracted from sperm, blood or tissue samples. This method was used for the genotyping of 273 hair follicle samples. When the DNA concentration was $>30 \mathrm{ng} / \mu \mathrm{l}$ all samples had the same high call rate ( > $99 \%$ ) as sperm samples confirming the robustness of this DNA extraction method for high throughput genotyping. Our data also establishes the suitability of the PorcineSNP60 BeadChip for genotyping the Finnish Landrace population.

Key-words: DNA extraction, Finnish Landrace, genotyping, hair follicles, pig, SNP 
Sironen, A. et al. High throughput genotyping from porcine hair follicles

\section{Introduction}

High throughput single-nucleotide polymorphism (SNP) genotyping methods are becoming increasingly important in population (Decker et al. 2009, Vonholdt et al. 2010) and association studies (Goddard and Hayes 2009) and also for genomic selection (Meuwissen et al. 2001). High throughput genotyping is now feasible due to the large number of SNP discovered by genome sequencing of various species and the development of new methods to efficiently genotype a large numbers of SNPs (Hayes et al. 2009). Genomic selection is based on a large reference population, in which animals are both phenotyped and genotyped. Previously collected samples from a reference population with reliable breeding values are required to predict genomic breeding values (GEBV) in subsequent generations. Similarly, existing samples can be used for the analysis of population diversity and identification of disease associated genomic regions by high throughput genotyping. DNA extracted from sperm, blood or tissue samples can be used for high quality genotyping using chip technology (Li et al. 2008, Jiang et al. 2010), but invasive procedures are required to collect such samples causing unnecessary pain and distress for sampled animals. Furthermore, these samples are also expensive and time consuming to collect.

The hair root is known to contain DNA and therefore represents a non-invasive source of DNA. The collection, transportation and storage of hair samples do not require any special procedures and as a result offers a painless and inexpensive alternative to the sampling of other tissues. Several methods have been described for polymerase chain reaction (PCR) amplification of single markers from hair samples (Amendola-Pimenta et al. 2009, Hayashida et al. 2009, Sironen et al. 2010). However, the low quality of DNA obtained has prevented the use of these samples for high throughput genotyping. In this study, we have assessed several possible methods for DNA preparation from porcine (Sus scrofa) hair roots for genotyping with the chip technology. Based on our analysis we have developed a reliable method for the preparation of
DNA from the hair root for genotyping with the PorcineSNP60 Genotyping BeadChip (Illumina).

\section{Material and Methods}

\section{DNA samples}

Genomic DNA was prepared from porcine (Finnish Landrace) hair roots collected during years 2000-2006 (samples of 273 boars were used for this study) and stored at room temperature. Control DNA was extracted from sperm samples using a phenol/ chloroform extraction protocol. For the assessment of various DNA preparation methods replicate of samples $(n>4)$ including the hair follicle of 5-15 hairs were used:

1. The hair roots were lysed in lysis buffer [proteinase $\mathrm{K} 0.5 \mathrm{mg} / \mathrm{ml}$ and $2 \mu 1 \mathrm{Mg}$-free PCR Buffer (Dynazyme DNA polymerase, Finnzymes) in $\mathrm{dH}_{2} \mathrm{O}$ ] at $55^{\circ} \mathrm{C}$ for $60 \mathrm{~min}$ following Proteinase $\mathrm{K}$ inactivation at $98^{\circ} \mathrm{C}$ for $10 \mathrm{~min}$.

2. DNA purification from the lysed samples (first preparation method) by ethanol (EtOH) precipitation with $100 \%$ ethanol and sodium acetate (0.3M, pH 5.2) at $-20^{\circ} \mathrm{C}$ for $30 \mathrm{~min}$ following centrifugation at $13000 \mathrm{rpm}$ for $10 \mathrm{~min}$. Any residual salt was washed with $70 \%$ ethanol and centrifuged at $13000 \mathrm{rpm}$ for $10 \mathrm{~min}$. Precipitated DNA was air dried and dissolved in $20 \mu \mathrm{l}$ of distilled $\mathrm{H}_{2} \mathrm{O}$.

3. DNA extraction from the lysed samples (first preparation method) by phenol/chloroform.

4. DNA purification from the lysed samples (first preparation method) by InstaGene matrix (BioRad) following the protocol supplied by the manufacture.

5. Hair roots were lysed in ATL lysis buffer (Qiagen) with proteinase $\mathrm{K}(10 \mathrm{mg} / \mathrm{ml})$ at $55^{\circ} \mathrm{C}$ for 60 min following Proteinase $\mathrm{K}$ inactivation at $98^{\circ} \mathrm{C}$ for $10 \mathrm{~min}$. Thereafter the DNA was purified with ethanol precipitation.

6. Hair roots were lysed in lysis buffer $(10 \mathrm{mM}$ Tris $\mathrm{HCl}, \mathrm{pH} 8.0,100 \mathrm{mM} \mathrm{NaCl}, 1 \mathrm{mM}$ EDTA, $0.5 \% \mathrm{SDS})$ with proteinase $\mathrm{K}(10 \mathrm{mg} / \mathrm{ml})$ at $55^{\circ} \mathrm{C}$ for $60 \mathrm{~min}$ following Proteinase $\mathrm{K}$ inactivation at 
Vol. 20(2011): 143-150.

$98^{\circ} \mathrm{C}$ for $10 \mathrm{~min}$. Thereafter, DNA was purified by precipitation with ethanol.

7. Extraction of DNA with DNeasy Blood \& Tissue Kit (Qiagen) according to the instructions supplied by the manufacturer.

For the lysis protocols $(1,5-6)$ the effect of addition of $\mathrm{MgCl}_{2}(2 \mathrm{mM})$ and DTT $(100 \mu \mathrm{M})$ and dissolution of the hair root by mechanical force (with a rod or FastPrep homogenization system) were also tested. The concentration of extracted DNA was measured with a Nanodrop spectrophotometer (NanoDrop Technologies) and a Qubit Quantitation Platform (Invitrogen). Nanodrop was also used for analysing the purity of extracted DNA. Absorbance at $260 \mathrm{~nm}$ quantified the amount of DNA and protein contamination was detected at $280 \mathrm{~nm}$. The ratio of absorbance at $\mathrm{A} 260 / 280$ and $\mathrm{A} 230 / 260$ was used to determine the purity of DNA samples. The Qubit platform uses fluorescence-based QuantiTTM assays, where fluorescence label binds specifically to DNA. Although the specificity to dsDNA increases the accuracy of the DNA concentration, this detection system does not give any information about the purity of the sample. The accuracy of these concentration measurements were also confirmed by quantitative PCR (qPCR).

The qPCR was performed with an ABI 7000 Sequence Detection System in 96-well microtiter plates using Absolute qPCR SYBR Green ROX Mix (VWR). DNA was amplified using primers for the microsatellite marker SW2411 (Forward CCTGGACTCATTCTTGCTTTG, reverse TTCCTATTCTGTCCTGCCTTG). Amplification by qPCR contained $12.5 \mu$ of Absolute qPCR SYBR Green Mix, $20 \mathrm{ng}$ of DNA, and $70 \mathrm{nM}$ of each primer in a final volume of $25 \mu 1$. Amplifications were initiated with 15 min enzyme activation at $95{ }^{\circ} \mathrm{C}$ followed by 40 cycles of denaturation at $95^{\circ} \mathrm{C}$ for $15 \mathrm{~s}$, primer annealing at $60^{\circ} \mathrm{C}$ for $30 \mathrm{~s}$, and extension at 72 ${ }^{\circ} \mathrm{C}$ for $30 \mathrm{~s}$. All samples were amplified in duplicate and a water sample was used as a negative control. A standard curve was produced by serial dilutions of DNA extracted from boar sperm (quantified with Nanodrop spectrophotometer). Quantities of DNA in the sample were estimated from the standard curve. Raw data were analyzed with the sequence detection software (Applied Biosystems).

\section{Genotyping}

For high throughput genotyping selected samples were analyzed on the PorcineSNP60 Genotyping BeadChip (Illumina Ltd) in the Institute for Molecular Medicine Finland (FIMM). The PorcineSNP60 BeadChip has recently been developed as an outcome from the porcine whole genome sequencing project (Ramos et al. 2009). The concentration of samples analyzed by the BeadChip was estimated by Qubit measurements and the purity was confirmed by Nanodrop. The concentration varied between $2-100 \mathrm{ng} / \mu 1$.

DNA samples of three boars with three different DNA preparation methods (1,2 and 7) from hair roots and a reference sample extracted from sperm were selected for genotyping with the PorcineSNP60 BeadChip. Furthermore, a duplicate sample of DNA from boar sperm was included on the chip in order to compare the differences amongst identical sperm samples and variation between DNA preparation methods and sperm samples. Based on these analyses the most reliable DNA extraction method for hair samples was selected and an additional 280 samples were analyzed with the PorcineSNP60 BeadChip.

\section{Statistical analysis}

The effect of DNA concentration and genotyping batch on call rate was evaluated by multivariate ANOVA-method (SAS Enterprise Guide 4.3, SAS Institute Inc.).

\section{Results and discussion}

\section{Evaluation of DNA preparation methods}

A wide range of DNA preparation methods were tested including a basic lysis protocol with different lysis buffers. The hair root lysis has been successfully implemented in single marker analysis (Sironen 
Sironen, A. et al. High throughput genotyping from porcine hair follicles

et al. 2010), however the purity is extremely low in these samples. No clear differences in DNA yield or purity were detected between lysis buffers (methods 2, 5 and 6) or following modification of the lysis protocol (addition of DTT/ $\mathrm{MgCl}_{2}$ or dissolution, Table 1). The most consistent results were produced using 15 hair roots, even though 10 or even 5 hair roots yielded adequate amounts of DNA depending on the sample (data not shown). Therefore the lysis buffer (method 1) used in our previous studies (Sironen et al. 2010) with 15 hair roots was selected for further purification.

DNA extraction with the InstaGene matrix (method 4) and by chloroform/phenol (method 3) resulted in low DNA concentrations (Table 1). Ethanol precipitation (method 2 ) and a commercial extraction kit (method 7) yielded relatively high amounts of good quality DNA (Table 1). Thus, for high throughput genotyping, DNA extracted from samples using methods 2 and 7 were selected. In addition, samples prepared by lysis (method 1) were also genotyped, since the concentration of DNA was assumed to be highest in these samples although the purity was low and interfered with the determination of DNA concentration by Qubit and Nanodrop.

\section{Comparison of different concentration assays}

The concentration of DNA in various preparations was evaluated with the Nanodrop spectrophotometer and Qubit platform. Concentrations of DNA were found to be approximately 10 times higher based on the Nanodrop than Qubit analysis (Table 1). Therefore, the determination of DNA concentration was evaluated further by qPCR and a standard curve prepared from DNA extracted from boar sperm (quantified with Nanodrop spectrophotometer). The same amount (20 ng) of DNA based on each concentration assay was added to the qPCR amplification and the concentration was compared to the standard curve. Qubit measurements appeared to be consistent with the results from qPCR and were therefore used to evaluate the concentration of DNA for chip genotyping.

Table 1. Protocols tested for the preparation of total DNA from porcine hair roots. Results from two different concentration measurement methods are presented. Sample purity (260/280 and 260/230 ratios) was assessed by Nanodrop.

\begin{tabular}{|c|c|c|c|c|}
\hline Protocol (n) & $\begin{array}{l}\text { Nanodrop ng/ } \mu \mathrm{l} \\
\quad(\text { mean, SD) }\end{array}$ & $\begin{array}{c}260 / 280 \\
\text { ratio }\end{array}$ & $260 / 230$ ratio & 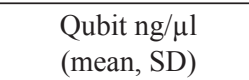 \\
\hline 1. Lysis (10) & $58-500(202,157)$ & $<1$ & $<0.5$ & $0.9-29(12,11.7)$ \\
\hline 2.Lysis + EtOH precipitation (36) & $15-500(168,137)$ & $1.8-2$ & $0.9-1.5$ & $1.5-100(21,17)$ \\
\hline 3.Lysis+phenol/chloroform (3) & $22-47(36,12.5)$ & $1.8-1.9$ & 2 & - \\
\hline 4. Lysis+BioRad matrix (5) & $35-83(58,24)$ & $1.3-1.7$ & 0.8 & $0.5-3(1.8,1.2)$ \\
\hline 5. ATL Qiagen lysis (5) & $2.5-21.7(11,8)$ & $<1$ & $<0.6$ & $0.2-0.5(0.35,0.12)$ \\
\hline 6. SDS+EDTA lysis (5) & $2-22(12,8.5)$ & $<1$ & $<0.5$ & $0.14-0.5(0.3,0.15)$ \\
\hline 7. Qiagen kit extraction (28) & $32-450(144,139)$ & $1.8-2$ & $0.5-1.8$ & $2-100(38,26)$ \\
\hline
\end{tabular}

$\mathrm{n}=$ number of samples 
Vol. 20(2011): 143-150.

\section{SNP quality measures of the Porcine- SNP60 Genotyping BeadChip}

The total number of SNPs in the PorcineSNP60 BeadChip is 62163. However, 2815 SNPs did not work for any of the genotyped samples and 9216 SNPs were monomorphic in the data set of Finnish Landrace pigs. Excluding those SNPs, the average minor allele frequency was $0.25(\mathrm{SD}=0.14)$. Furthermore, the observed distribution of P-values of the Hardy-Weinberg equilibrium test statistic did not differ from expectations; 183 SNPs (excluding the SNPs on the X-chromosome) had $p$-values lower than $1.0 \mathrm{E}-06$ which is much less than could be expected by chance. Thus, the PorcineSNP60 BeadChip appears to be a robust and reliable method for genotyping of Finnish Landrace pigs.

\section{Quality of the PorcineSNP60 BeadChip genotyping results with hair root sam- ples}

The comparison of the genotyping results from three different DNA preparation methods illustrated the importance of the quality of analysed DNA samples. The lowest call rate (CR, 90-93\%, Table 2) was detected with the unpurified lysis samples (method $1)$. When compared with the sperm sample, the percentage of missing alleles was $2-5 \%$ and the percentage of different allele calls (e.g. A in one sample and $\mathrm{G}$ in the other) was $0.09-0.31 \%$ (Table 3 ). These differences substantially reduce the reliability of genotypes produced from lysed hair root samples. Samples of DNA extracted by the ethanol precipitation (method 2) and Qiagen purification (method 7) methods showed high call rates; 94-95 $\%$ and $95 \%$ for methods 2 and 7 , respectively (Table 2 ) and very low percentage of differences when compared with sperm samples (Table 3). These values are consistent with the differences seen in duplicate DNA samples extracted from sperm (Table 3). Excluding the $2815 \mathrm{SNPs}$, that did not work for any of the genotyped samples, the average CR
Table 2. The overall call rate (CR) for three samples (13) prepared by different DNA extraction methods and a control sample (4a and $4 \mathrm{~b}$ ) after Illumina Beadchip genotyping. The corrected $\mathrm{CR}$ indicates the $\mathrm{CR}$ after excluding SNPs that did not work for any of the samples analyzed. The number or letter listed in the method column indicates the DNA preparation method: $S=$ DNA extracted from sperm, $1=$ lysis, $2=1$ ysis + EtOH precipitation and 7=Qiagen kit.

\begin{tabular}{llll}
\hline Sample & Method & CR & CR corrected \\
\hline 1 & $\mathrm{~S}$ & 0.950 & 0.995 \\
1 & 1 & 0.901 & 0.944 \\
1 & 7 & 0.950 & 0.995 \\
1 & 2 & 0.940 & 0.985 \\
2 & $\mathrm{~S}$ & 0.950 & 0.995 \\
2 & 1 & 0.931 & 0.975 \\
2 & 7 & 0.950 & 0.995 \\
2 & 2 & 0.950 & 0.994 \\
3 & $\mathrm{~S}$ & 0.950 & 0.995 \\
3 & 1 & 0.913 & 0.956 \\
3 & 7 & 0.950 & 0.995 \\
3 & 2 & 0.937 & 0.981 \\
$4 \mathrm{a}$ & $\mathrm{S}$ & 0.950 & 0.994 \\
$4 \mathrm{~b}$ & $\mathrm{~S}$ & 0.950 & 0.994 \\
\hline
\end{tabular}

for all Qiagen and EtOH precipitated samples was $0.9982(\mathrm{SD}=0.007)$.

Additional extractions of DNA were performed using the Qiagen Blood and Tissue kit (method 7), since the overall call rates were slightly higher in these samples compared with EtOH precipitated samples. However, the EtOH precipitation from hair root lyses appears to be a feasible method for DNA preparation for high throughput genotyping. The CR for 231/270 (86 \%) additional hair root samples was $>99 \%$ (after exclusion of SNPs that did not work in any samples). For 28 samples the call rate was $>90 \%$, for 18 samples $>72 \%$ and three samples did not work. The purity (260/280 ratio) was $>1.7$ for all genotyped samples and did not explain the lower call rates, but the DNA concentration of the samples varied between 2-100 $\mathrm{ng} / \mu \mathrm{l}$. Samples with a lower DNA concentration were also genotyped, because for some hair root samples it was extremely difficult or even impossible to obtain a sufficiently high DNA concentra- 
Sironen, A. et al. High throughput genotyping from porcine hair follicles

Table 3. Differences in allele calls after Beadchip genotyping of hair root samples prepared by various DNA extraction methods. Missing genotypes: number of genotypes that are missing in the comparison between hair and sperm samples. Different genotypes: number of genotypes that are different in the comparison between hair and sperm samples. The letter or number after the identification number indicates the DNA preparation method: $\mathrm{S}=\mathrm{DNA}$ extracted from sperm, $1=1 \mathrm{ysis}$, 2=lysis + EtOH precipitation and 7=Qiagen kit.

\begin{tabular}{lccccc}
\hline & $\begin{array}{c}\text { Missing } \\
\text { genotypes }\end{array}$ & $\%$ & $\begin{array}{c}\text { Different } \\
\text { genotypes }\end{array}$ & $\%$ \\
\hline PIG.1S & PIG.1_1 & 3068 & 4.94 & 193 & 0.31 \\
PIG.1S & PIG.1_7 & 48 & 0.08 & 32 & 0.05 \\
PIG.1S & PIG.1_2 & 618 & 0.99 & 38 & 0.06 \\
PIG.1_7 & PIG.1_2 & 604 & 0.97 & 71 & 0.11 \\
PIG.2S & PIG.2_1 & 1197 & 1.93 & 53 & 0.09 \\
PIG.2S & PIG.2_7 & 18 & 0.03 & 19 & 0.03 \\
PIG.2S & PIG.2_2 & 69 & 0.11 & 18 & 0.03 \\
PIG.2_7 & PIG.2_2 & 63 & 0.10 & 25 & 0.04 \\
PIG.3S & PIG.3_1 & 2301 & 3.70 & 157 & 0.25 \\
PIG.3S & PIG.3_7 & 18 & 0.03 & 37 & 0.06 \\
PIG.3S & PIG.3_2 & 822 & 1.32 & 57 & 0.09 \\
PIG.3_7 & PIG.3_2 & 816 & 1.31 & 44 & 0.07 \\
PIG.4aS & PIG.4bS & 64 & 0.10 & 52 & 0.08 \\
\hline
\end{tabular}

tion $(>50 \mathrm{ng} / \mu \mathrm{l})$. Thus, with the aim of genotyping a large population of animals with minimal effort the range in DNA concentration was considered acceptable. When the call rates were analysed in groups based on the DNA concentration, a clear association between concentration and CR was identified (Fig. 1). With low DNA concentrations $(<$ $30 \mathrm{ng} / \mu \mathrm{l})$ the proportion of samples with adequate call rates was lower than when the concentration exceeded $30 \mathrm{ng} / \mu \mathrm{l}$. Based on pair-wise comparisons using the Tukey's test there was a significant difference between sperm samples and hair samples when DNA concentration fell below $30 \mathrm{ng} /$ $\mu 1$, but no difference when the concentration was higher than $30 \mathrm{ng} / \mu 1$ (Table 4). Significant differences were also found between batch number four and batch number three (Table 4). Part of this may be due to the age and storage conditions of the hair samples, but some variation may also arise from handling of the sample during DNA extraction protocol. The acceptable limit for DNA concen-

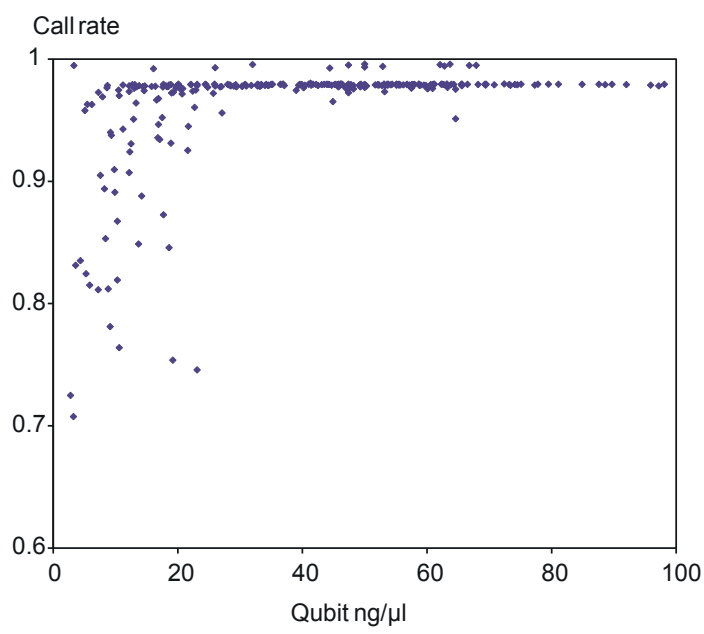

Fig. 1. Distribution plot of call rates against DNA concentration of hair root samples. At low concentrations the proportion of samples with an adequate call rate is lower than with samples containing DNA concentration above $30 \mathrm{ng} / \mu \mathrm{l}$. 
Vol. 20(2011): 143-150.

\begin{tabular}{|c|c|c|c|c|c|c|}
\hline DNA source & Class & $\mathrm{n}$ & Mean & $\mathrm{SD}$ & LS mean & $p$-value \\
\hline Hair root & $<10$ & 24 & 0.885 & 0.084 & 0.900 & $<.0001$ \\
\hline Hair root & $10-30$ & 80 & 0.953 & 0.052 & 0.969 & 0.005 \\
\hline Hair root & $30-50$ & 71 & 0.979 & 0.004 & 0.994 & 1 \\
\hline Hair root & $50-100$ & 98 & 0.980 & 0.005 & 0.994 & 1 \\
\hline Sperm & & 130 & 0.993 & 0.007 & 0.995 & \\
\hline \multirow[t]{4}{*}{ DNA batch } & 1 & 21 & 1.000 & 0.0002 & 0.975 & 0.099 \\
\hline & 2 & 87 & 0.995 & 0.0003 & 0.970 & 0.103 \\
\hline & 3 & 16 & 0.990 & 0.0139 & 0.981 & 0.003 \\
\hline & 4 & 279 & 0.963 & 0.0453 & 0.954 & \\
\hline Overall & & 403 & 0.973 & 0.041 & & \\
\hline
\end{tabular}

tration appeared to be $30 \mathrm{ng} / \mu 1$ producing $\mathrm{CR}>99$ $\%$ corresponding to the CR with DNA extracted from sperm or blood. However, samples with lower concentration of DNA also exhibited high call rates indicating that some deviation can be tolerated for successful genotyping. Therefore, depending on the experimental design, it may be necessary to increase sample numbers by decreasing the DNA concentration streshold.

\section{Conclusion}

Our results show that DNA from porcine hair roots using an appropriate extraction method generates reliable genotyping results with the Illumina PorcineSNP60 Genotyping BeadChip. These data allow a straightforward and inexpensive means of genotyping previously collected samples and/or large animal populations. The concentration of DNA appears to be the limiting factor in genotyping DNA from hair roots and can be used to confirm the high CRs. However, if the genotyping of large populations is required analysis of samples containing lower DNA concentrations may remain viable. The Qubit platform appeared to be a valid method for the measurement of DNA concentration. Furthermore, the SNPs on PorcineSNP60 BeadChip were highly polymorphic in the Finnish Landrace population highlighting the feasibility of this technology for genotyping of Finnish Landrace pigs.

Acknowledgements. Funding for this study was provided by the Finnish Ministry of Agriculture and Forestry (Makera). The assistance of Tiina Jaakkola and Tarja Hovivuori in DNA extraction and Päivi Lahermo (Institute for Molecular Medicine Finland, FIMM) in genotyping with PorcineSNP60 Genotyping BeadChip (Illumina) is greatly appreciated. 


\section{AGRICULTURAL AND FOOD SCIENCE}

Sironen, A. et al. High throughput genotyping from porcine hair follicles

\section{References}

Amendola-Pimenta, M., Garcia-Feria, L., Serio-Silva, J.C. \& Rico-Gray, V. 2009. Noninvasive collection of fresh hairs from free-ranging howler monkeys for DNA extraction. American Journal of Primatology 71: 359-363.

Decker, J.E., Pires, J.C., Conant, G.C., McKay, S.D., Heaton, M.P., Chen, K., Cooper, A., Vilkki, J., Seabury, C.M., Caetano, A.R., Johnson, G.S., Brenneman, R.A., Hanotte, O., Eggert, L.S., Wiener, P., Kim, J.J., Kim, K.S., Sonstegard, T.S., Van Tassell, C.P., Neibergs, H.L., McEwan, J.C., Brauning, R., Coutinho, L.L., Babar, M.E., Wilson, G.A., McClure, M.C., Rolf, M.M., Kim, J., Schnabel, R.D. \& Taylor, J.F. 2009. Resolving the evolution of extant and extinct ruminants with high-throughput phylogenomics. Proceedings of the National Academy of Sciences of the United States of America 106: 18644-18649.

Goddard, M.E. \& Hayes, B.J. 2009. Mapping genes for complex traits in domestic animals and their use in breeding programmes. Nature Reviews. Genetics 10: 381-391.

Hayashida, M., Iwao-Koizumi, K., Murata, S. \& Kinoshita, K. 2009. Single-tube genotyping from a human hair root by direct PCR. Analytical Sciences: The International Journal of the Japan Society for Analytical Chemistry 25: 1487-1489.

Hayes, B.J., Bowman, P.J., Chamberlain, A.J. \& Goddard, M.E. 2009. Invited review: Genomic selection in dairy cattle: progress and challenges. Journal of Dairy Science 92: 433-443.

Jiang, L., Liu, J., Sun, D., Ma, P., Ding, X., Yu, Y. \& Zhang, Q. 2010. Genome wide association studies for milk production traits in Chinese Holstein population. PloS One 5: e13661.
Li, J.Z., Absher, D.M., Tang, H., Southwick, A.M., Casto, A.M., Ramachandran, S., Cann, H.M., Barsh, G.S., Feldman, M., Cavalli-Sforza, L.L. \& Myers, R.M. 2008. Worldwide human relationships inferred from genomewide patterns of variation. Science (New York, N.Y.) 319: 1100-1104.

Meuwissen, T.H., Hayes, B.J. \& Goddard, M.E. 2001. Prediction of total genetic value using genome-wide dense marker maps. Genetics 157: 1819-1829.

Ramos, A.M., Crooijmans, R.P., Affara, N.A., Amaral, A.J., Archibald, A.L., Beever, J.E., Bendixen, C., Churcher, C., Clark, R., Dehais, P., Hansen, M.S., Hedegaard, J., Hu, Z.L., Kerstens, H.H., Law, A.S., Megens, H.J., Milan, D., Nonneman, D.J., Rohrer, G.A., Rothschild, M.F., Smith, T.P., Schnabel, R.D., Van Tassell, C.P., Taylor, J.F., Wiedmann, R.T., Schook, L.B. \& Groenen, M.A. 2009. Design of a high density SNP genotyping assay in the pig using SNPs identified and characterized by next generation sequencing technology. PloS One 4: e6524.

Sironen, A.I., Uimari, P., Serenius, T., Mote, B., Rothschild, M. \& Vilkki, J. 2010. Effect of polymorphisms in candidate genes on reproduction traits in Finnish pig populations. Journal of Animal Science 88: 821-827.

Vonholdt, B.M., Pollinger, J.P., Lohmueller, K.E., Han, E., Parker, H.G., Quignon, P., Degenhardt, J.D., Boyko, A.R., Earl, D.A., Auton, A., Reynolds, A., Bryc, K., Brisbin, A., Knowles, J.C., Mosher, D.S., Spady, T.C., Elkahloun, A., Geffen, E., Pilot, M., Jedrzejewski, W., Greco, C., Randi, E., Bannasch, D., Wilton, A., Shearman, J., Musiani, M., Cargill, M., Jones, P.G., Qian, Z., Huang, W., Ding, Z.L., Zhang, Y.P., Bustamante, C.D., Ostrander, E.A., Novembre, J. \& Wayne, R.K. 2010. Genomewide SNP and haplotype analyses reveal a rich history underlying dog domestication. Nature 8: 98-899-902. 